\title{
LOVE'S INTEGRAL AND OTHER RELATIONS BETWEEN SOLUTIONS TO MIXED BOUNDARY-VALUE PROBLEMS IN POTENTIAL THEORY
}

\author{
A. H. ENGLAND
}

(Received 5 February 1980)

\begin{abstract}
In a paper published in 1949, E. R. Love [10] found an integral relation between a separated solution (in oblate spheroidal coordinates) to a particular mixed boundaryvalue problem and a solution to the same problem using an integral representation. This note examines further representations of the same type which occur in some simple twoand three-dimensional potential problems.
\end{abstract}

\section{Introduction}

In two recent papers [5], [4], separated solutions of Laplace's equation in two and three dimensions were used to derive solutions to frictionless punch and crack problems in the linear theory of elasticity. In particular, quantities of physical interest, such as the contact stress under a punch or the displacement over a crack, could be expressed in terms of Chebyshev's polynomials in two-dimensional problems and Legendre polynomials in three-dimensional problems. There are several different methods of solution of the simplest mixed boundary-value problems for Laplace's equation and these yield solutions in different functional forms. In Section 2 the method of separation of variables is compared with the integral representation approach due to E. T. Copson [1], E. R. Love [10] and A. E. Green [6]. In this section, Love's integral representation is given and certain useful formulae derived from it. A comparison with the solutions obtained in terms of the appropriate Green's functions is made in Section 3.

Copyright Australian Mathematical Society 1981 
In Section 4, the same method is employed on plane problems and the separated solution compared with an integral representation due to England and Green [3]. In this case integral representations involving both Chebyshev and Legendre polynomials are found. Some relations connecting the separated solution with solutions obtained by alternative methods are also given.

\section{Axially-symmetric problems}

If $(r, \phi, z)$ are cylindrical polar coordinates, then the set of oblate spheroidal coordinates $(\eta, \xi, \phi)$ are related to the cylindrical polar coordinates by

$$
r=\left\{\left(1-\eta^{2}\right)\left(1+\xi^{2}\right)\right\}^{1 / 2} \text { and } z=\eta \xi
$$

where $-1 \leqslant \eta \leqslant 1$ and $\xi \geqslant 0$. The surfaces $\xi=$ constant are oblate spheroids whose axis of revolution is the $z$-axis. The surface $\xi=0, \eta \geqslant 0$ is the upper face of the $\operatorname{disc} z=0,0 \leqslant r \leqslant 1$. The surfaces $\eta=$ constant are hyperboloids of revolution about the $z$-axis. The half-space $z \geqslant 0$ corresponds to $0 \leqslant \eta \leqslant 1$ and $\eta=0$ is the region $z=0, r \geqslant a$.

In oblate spheroidal coordinates, separated solutions of Laplace's equation which vanish as $r^{2}+z^{2} \rightarrow \infty$ are

$$
P_{n}(\eta) Q_{n}(i \xi)
$$

where $P_{n}$ is the Legendre polynomial of degree $n$ and $Q_{n}$ is the Legendre function of the second kind. Note that $Q_{n}(\zeta)$, where $\zeta$ is a complex variable, is defined in the complex $\zeta$-plane cut along the interval $\operatorname{Im} \zeta=0,|\operatorname{Re} \zeta|<1$.

These separated solutions lead naturally to the following mixed boundaryvalue problems for the harmonic functions $\chi$ and $\psi$ defined in the half-space $z \geqslant 0$ subject to the stated conditions on $z=0$.

\section{Problem A}

$$
\left.\begin{array}{c}
\text { On } z=0, \chi=P_{2 n}\left\{\left(1-r^{2}\right)^{1 / 2}\right\} \text { for } 0 \leqslant r \leqslant 1, \text { and } \\
\frac{\partial \chi}{\partial z}=0 \text { for } r>1 .
\end{array}\right\}
$$

Solution:

$$
\chi=P_{2 n}(\eta) Q_{2 n}(i \xi) / Q_{2 n}(i 0+)
$$

where

$$
Q_{2 n}(i 0+)=\pi P_{2 n}(0) / 2 i .
$$

Problem $A$ corresponds to a charged disc problem in electrostatics and a contact problem in elasticity. 


\section{Problem B}

On $z=0$,

and

$$
\left.\begin{array}{c}
\frac{\partial \psi}{\partial z}=\frac{P_{2 n+1}\left\{\left(1-r^{2}\right)^{1 / 2}\right\}}{\left(1-r^{2}\right)^{1 / 2}} \text { for } 0 \leqslant r \leqslant 1, \\
\psi=0 \text { for } r \geqslant 1 .
\end{array}\right\}
$$

Solution:

$$
\psi=P_{2 n+1}(\eta) Q_{2 n+1}(i \xi) / i Q_{2 n+1}^{\prime}(i 0+)
$$

where

$$
i Q_{2 n+1}^{\prime}(i 0+)=\pi(2 n+1) P_{2 n}(0) / 2
$$

Problem $B$ corresponds to the symmetrical inflation of a penny-shaped crack in elasticity.

Problem $A$ may be reduced to an Abel integral equation by using the integral representation due to Copson [1], Love [10] and Green [6]:

$$
\chi(r, z)=\operatorname{Re} \int_{0}^{1} \frac{g(t)}{\left\{r^{2}+(z+i t)^{2}\right\}^{1 / 2}} d t .
$$

This representation automatically satisfies the second boundary condition of (3) and the first condition becomes

$$
\int_{0}^{r} \frac{g(t)}{\left(r^{2}-t^{2}\right)^{1 / 2}} d t=P_{2 n}\left\{\left(1-r^{2}\right)^{1 / 2}\right\} \quad \text { for } 0 \leqslant r<1 .
$$

This Abel integral equation may be inverted to give

$$
g(t)=\frac{2}{\pi} \frac{d}{d t} \int_{0}^{t} \frac{r P_{2 n}\left\{\left(1-r^{2}\right)^{1 / 2}\right\}}{\left(t^{2}-r^{2}\right)^{1 / 2}} d r .
$$

This integral indicates that $g(t)$ is a polynomial of degree $2 n$ in $t$. The integral has been evaluated by England and Shail [4] using the addition theorem for the Legendre polynomials and yields the surprisingly simple result

$$
g(t)=\frac{2}{\pi} \frac{P_{2 n}(t)}{P_{2 n}(0)}
$$

Hence there are two representations for the unique harmonic function $\chi$, namely (4) and (9) in which $g(t)$ is given by (12). Equating these solutions yields the identity

$$
i P_{2 n}(\eta) Q_{2 n}(i \xi)=\operatorname{Re} \int_{0}^{1} \frac{P_{2 n}(t)}{\left\{r^{2}+(z+i t)^{2}\right\}^{1 / 2}} d t
$$


This identity was originally established by E. R. Love [10] and will be referred to as Love's integral.

The corresponding identity for the odd-order functions is

$$
P_{2 n+1}(\eta) Q_{2 n+1}(i \xi)=\operatorname{Im} \int_{0}^{1} \frac{P_{2 n+1}(t)}{\left\{r^{2}+(z+i t)^{2}\right\}^{1 / 2}} d t .
$$

This leads directly to the solution of Problem $B$. In this case the corresponding form of the integral representation of Copson, Love and Green is

$$
\psi=\operatorname{Im} \int_{0}^{1} \frac{k(t)}{\left\{r^{2}+(z+i t)^{2}\right\}^{1 / 2}} d t
$$

and this automatically satisfies the second boundary condition of (6). The first boundary condition of (6) yields the Abel integral equation

$$
\int_{0}^{r} \frac{k^{\prime}(t)}{\left(r^{2}-t^{2}\right)^{1 / 2}} d t=\frac{1}{\left(1-r^{2}\right)^{1 / 2}} P_{2 n+1}\left\{\left(1-r^{2}\right)^{1 / 2}\right\}, \quad 0 \leqslant r \leqslant 1,
$$

which may be inverted to give

$$
k(t)=\frac{2}{\pi} \int_{0}^{t} \frac{r P_{2 n+1}\left\{\left(1-r^{2}\right)^{1 / 2}\right\}}{\left(t^{2}-r^{2}\right)^{1 / 2}\left(1-r^{2}\right)^{1 / 2}} d r .
$$

Hence $k(t)$ is a polynomial of degree $2 n+1$ in $t$. On comparing (15) with the separated solution (7) and the second Love integral (14), we see that

$$
k(t)=\frac{2 P_{2 n+1}(t)}{\pi(2 n+1) P_{2 n}(0)} .
$$

A number of useful formulae spring from the identities (13) and (14) and involve Abel transforms of the Legendre functions. Some of the following relations were given by England and Shail [4].

If we use the notation

$$
A\{f(t), x\}=\frac{2}{\pi} \int_{0}^{x} \frac{f(t)}{\left(x^{2}-t^{2}\right)^{1 / 2}} d t=j(x),
$$

then the inverse Abel transformation is given by

$$
f(t)=A^{-1}\{j(x), t\}=\frac{d}{d t} \int_{0}^{t} \frac{x j(x)}{\left(t^{2}-x^{2}\right)^{1 / 2}} d x=\frac{\pi}{2} \frac{d}{d t} A\{x j(x), t\} .
$$

Note also that on making the substitutions $t=\left(1-s^{2}\right)^{1 / 2}$ and $x=\left(1-r^{2}\right)^{1 / 2}$ the transform (19) becomes

$$
A\{f(t), x\}=\frac{2}{\pi} \int_{r}^{1} \frac{s f\left\{\left(1-s^{2}\right)^{1 / 2}\right\}}{\left(1-s^{2}\right)^{1 / 2}\left(s^{2}-r^{2}\right)^{1 / 2}} d s=j\left\{\left(1-r^{2}\right)^{1 / 2}\right\}
$$


The corresponding inversion formula is

$$
h(s)=\frac{s f\left\{\left(1-s^{2}\right)^{1 / 2}\right\}}{\left(1-s^{2}\right)^{1 / 2}}=-\frac{d}{d s} \int_{s}^{1} \frac{r j\left\{\left(1-r^{2}\right)^{1 / 2}\right\}}{\left(r^{2}-s^{2}\right)^{1 / 2}} d r .
$$

Using the obvious notation, if

$$
A_{1}\{h(s), r\}=\frac{2}{\pi} \int_{r}^{1} \frac{h(s)}{\left(s^{2}-r^{2}\right)^{1 / 2}} d s=k(r)
$$

then

$$
A_{1}^{-1}\{k(r), s\}=-\frac{\pi}{2} \frac{d}{d s} A_{1}\{r k(r), s\}
$$

The Legendre function relations which may be deduced from (13) and (14) are

$$
\begin{gathered}
A\left\{P_{2 n}(t), x\right\}=P_{2 n}(0) P_{2 n}\left\{\left(1-x^{2}\right)^{1 / 2}\right\} \\
\frac{d}{d x} A\left\{t P_{2 n}\left\{\left(1-t^{2}\right)^{1 / 2}\right\}, x\right\}=\frac{2 P_{2 n}(x)}{\pi P_{2 n}(0)}, \\
A_{1}\left\{\frac{t P_{2 n}\left\{\left(1-t^{2}\right)^{1 / 2}\right\}}{\left(1-t^{2}\right)^{1 / 2}}, x\right\}=P_{2 n}(0) P_{2 n}(x) \\
\frac{d}{d x} A_{1}\left\{t P_{2 n}(t), x\right\}=-\frac{2 x P_{2 n}\left\{\left(1-x^{2}\right)^{1 / 2}\right\}}{\pi P_{2 n}(0)\left(1-x^{2}\right)^{1 / 2}} \\
A_{1}\left\{P_{2 n+1}(t), x\right\}=\frac{2 P_{2 n+1}\left\{\left(1-x^{2}\right)^{1 / 2}\right\}}{\pi(2 n+1) P_{2 n}(0)} \\
A_{1}\left\{t P_{2 n+1}\left\{\left(1-t^{2}\right)^{1 / 2}\right\}, x\right\}=-(2 n+1) P_{2 n}(0) P_{2 n+1}(x), \\
A\left\{\frac{t P_{2 n+1}\left\{\left(1-t^{2}\right)^{1 / 2}\right\}}{\left(1-t^{2}\right)^{1 / 2}}, x\right\}=\frac{2 P_{2 n+1}(x)}{\pi(2 n+1) P_{2 n}(0)},
\end{gathered}
$$

and

$$
\frac{d}{d x} A\left\{t P_{2 n+1}(t), x\right\}=\frac{(2 n+1) P_{2 n}(0) x P_{2 n+1}\left\{\left(1-x^{2}\right)^{1 / 2}\right\}}{\left(1-x^{2}\right)^{1 / 2}}
$$

These relations hold with $x$ in the range $0<x<1$. 


\section{Integral transform techniques}

Hankel transform techniques may be applied to Problems $A$ and $B$. If we put

$$
\chi=\int_{0}^{\infty} A(\xi) J_{0}(\xi r) e^{-\xi z} d \xi,
$$

the boundary conditions (3) reduce to the dual integral equations

$$
\int_{0}^{\infty} A(\xi) J_{0}(\xi r) d \xi=P_{2 n}\left\{\left(1-r^{2}\right)^{1 / 2}\right\} \text { for } 0 \leqslant r<1,
$$

and

$$
\int_{0}^{\infty} \xi A(\xi) J_{0}(\xi r) d \xi=0 \text { for } r>1 .
$$

If we follow Sneddon's method [13, Section 4.4] and put

$$
A(\xi)=\int_{0}^{1} g(t) \cos (\xi t) d t,
$$

then the integral representation (9) results and the method of solution is identical to that described in Section 2 . Alternatively, if we denote $\partial \chi / \partial z$ by $\sigma(r)$ on $z=0$ for $0 \leqslant r \leqslant 1$, then

$$
A(\xi)=-\int_{0}^{1} s \sigma(s) J_{0}(\xi s) d s .
$$

When this form is substituted into the first condition of $(28)$, it is found that $\sigma(s)$ satisfies the singular integral equation

$$
\frac{2}{\pi} \int_{0}^{1} \frac{s}{(s+r)^{1 / 2}} K\left\{\frac{2(s r)^{1 / 2}}{s+r}\right\} \sigma(s) d s=P_{2 n}\left\{\left(1-r^{2}\right)^{1 / 2}\right\} \quad \text { for } 0 \leqslant r \leqslant 1 .
$$

In (29), $K(\xi)$ is a complete elliptic integral.

Since this problem has the known solution (4), it is straightforward to show that

$$
\sigma(s)=\frac{2}{\pi\left\{P_{2 n}(0)\right\}^{2}} \frac{P_{2 n}\left\{\left(1-r^{2}\right)^{1 / 2}\right\}}{\left(1-r^{2}\right)^{1 / 2}} \text { for } 0 \leqslant r<1 .
$$

This relation has been given by Popov [11], [12]. The complete solution to the problem when expressed in terms of Hankel transforms (or equivalently via the Green's function for a half-space) is then

$$
\begin{aligned}
\chi & =\int_{0}^{1} s \sigma(s) \int_{0}^{\infty} J_{0}(\xi r) J_{0}(\xi s) e^{-\xi z} d \xi d s \\
& =\frac{2}{\pi} \int_{0}^{1} \frac{s \sigma(s)}{\left\{(r+s)^{2}+z^{2}\right\}^{1 / 4}} K\left[\frac{2(r s)^{1 / 2}}{\left\{(r+s)^{2}+z^{2}\right\}^{1 / 2}}\right] d s .
\end{aligned}
$$


Hence there exists the identity that the representation (4) is also equal to (31) where $\sigma(s)$ is defined by (30). Popov [12] and Gladwell and England [5] have used these relations in the solution of certain contact problems.

Problem B may be solved in a similar fashion. If $\psi$ has the Hankel transform representation

$$
\psi=\int_{0}^{\infty} B(\xi) J_{0}(\xi r) e^{-\xi z} d \xi
$$

then the boundary conditions (6) reduce to the dual integral equations

$$
\int_{0}^{\infty} \xi B(\xi) J_{0}(\xi r) d \xi=-\frac{P_{2 n+1}\left\{\left(1-r^{2}\right)^{1 / 2}\right\}}{\left(1-r^{2}\right)^{1 / 2}} \text { for } 0 \leqslant r \leqslant 1,
$$

and

$$
\int_{0}^{\infty} B(\xi) J_{0}(\xi r) d \xi=0 \text { for } r>1
$$

If we put

$$
B(\xi)=-\int_{0}^{1} k(t) \sin (\xi t) d t,
$$

then the representation (15) results and the dual integral equations reduce to (16). Alternatively, if we denote $\psi(r, 0)$ by $\tau(r)$ in $0 \leqslant r \leqslant 1$, then

$$
B(\xi)=\xi \int_{0}^{1} s \tau(s) J_{0}(\xi s) d s .
$$

In this case the representation for $\psi$ is

$$
\psi=-\frac{2}{\pi} \frac{d}{d z} \int_{0}^{1} \frac{s \tau(s)}{\left\{(r+s)^{2}+z^{2}\right\}^{1 / 4}} K\left[\frac{2(r s)^{1 / 2}}{\left\{(r+s)^{2}+z^{2}\right\}^{1 / 2}}\right] d s .
$$

On comparison with Problem $B$, the value of $\tau(s)$ is

$$
-\frac{2}{\pi(2 n+1)^{2}\left\{P_{2 n}(0)\right\}^{2}} P_{2 n+1}\left\{\left(1-r^{2}\right)^{1 / 2}\right\} \text {. }
$$

Thus the integral (32) is equal to (7) for each integral value of $n$.

A different approach to these problems was adopted by Heins and MacCamy [7] and is based on earlier work by Erdelyi. Their method depends on the property that if $\phi(x, y)$ is symmetric about $y=0$ and $\phi(x, y)$ satisfies Laplace's equation in two dimensions, then the Abel transformation $A\{\phi(z, y), y \rightarrow r\}=$ $U(r, z)$ forms an axially-symmetric solution of Laplace's equation. There is a corresponding inverse transformation relating $\phi$ to $U(r, z)$. The method converts a mixed boundary-value problem for an axially-symmetric potential into a Dirichlet problem for a plane potential but does not appear to yield significant results when applied to Problems $A$ and $B$ of Section 2 or to the basic problems 
of the next section. In a later paper, Heins [8] represents an axially-symmetric potential by means of the Poisson integral representation

$$
U(r, z)=\frac{1}{\pi} \int_{0}^{\pi} U(0, z+i r \cos \theta) d \theta,
$$

and solves Problem $A$ in the case $n=0$ when the potential of the disc is constant. With care this method may be extended to rederive Love's integral formula (13).

\section{Plane problems}

Separated solutions of Laplace's equation in elliptical polar coordinates are most easily produced by using the conformal transformation

$$
z=\frac{1}{2}\left(\zeta+\frac{1}{\zeta}\right) \text { for }|\zeta| \geqslant 1 .
$$

This maps the region $|\zeta| \geqslant 1$ in the $\zeta$-plane onto the $z$-plane cut along $y=0$ for $|x| \leqslant 1$ where $z=x+i y$. The natural analytic function in the region $|\zeta| \geqslant 1$ is $\zeta^{-n}$ which, in terms of $z$, becomes

$$
R_{n}(z)=\left\{z-\left(z^{2}-1\right)^{1 / 2}\right\}^{n} .
$$

The function $R_{n}(z)$ is analytic in the cut $z$-plane. The real and imaginary parts of the function $R_{n}(z)$ satisfy the following fundamental mixed boundary-value problems for Laplace's equation in the half-plane $y>0$.

\section{Problem C}

The harmonic function $\phi_{n}=\operatorname{Re}\left\{R_{n}(z)\right\}$ satisfies the following boundary conditions on $y=0+$ :

and

$$
\left.\begin{array}{cc}
\phi_{n}=T_{n}(x) & \text { for }|x| \leqslant 1 \\
\partial \phi_{n} / \partial y=0 & \text { for }|x|>1,
\end{array}\right\}
$$

where $T_{n}(x)$ is a Chebyshev polynomial of the first kind. Also on $y=0+$,

and

$$
\left.\begin{array}{ll}
\partial \phi_{n} / \partial y=-n\left(1-x^{2}\right)^{-1 / 2} T_{n}(x) & \text { for }|x|<1 \\
\phi=\left\{x-\operatorname{sgn}(x)\left(x^{2}-1\right)^{1 / 2}\right\}^{n} & \text { for }|x|>1 .
\end{array}\right\}
$$


Problem $C$ corresponds to a contact problem in two-dimensional elasticity and a charged strip in electrostatics.

\section{Problem D}

The harmonic function $\theta_{n}=\operatorname{Im}\left\{R_{n}(z)\right\}$ satisfies the following boundary conditions on $y=0+$ :

and

$$
\left.\begin{array}{c}
\partial \theta_{n} / \partial y=n U_{n-1}(x) \text { for }|x| \leqslant 1 \\
\theta_{n}=0 \text { for }|x| \geq 1,
\end{array}\right\}
$$

where $U_{n}(x)$ is a Chebyshev polynomial of the second kind. Also on $y=0+$,

$$
\left.\begin{array}{c}
\theta_{n}=-\left(1-x^{2}\right)^{1 / 2} U_{n-1}(x) \quad \text { for }|x| \leqslant 1 \\
\partial \theta_{n} / \partial y=-\frac{n\left\{x-\operatorname{sgn}(x)\left(x^{2}-1\right)^{1 / 2}\right\}^{n}}{\operatorname{sgn}(x)\left(x^{2}-1\right)^{1 / 2}} \text { for }|x| \geqslant 1 .
\end{array}\right\}
$$

and

Problem $D$ corresponds to a plane crack problem in elasticity.

Alternatively these problems may be solved by use of the integral representation suggested by England and Green [3]. This is the two-dimensional analogue of the integral representation employed in Section 2. If we consider the anti-symmetrical solution given by Problem $C$ in which $n=2 k+1$ and $\phi_{2 k+1}$ $=\operatorname{Re}\left\{R_{2 k+1}(z)\right\}$, then an alternative representation for $\phi_{2 k+1}$ is

$$
\phi_{2 k+1}=\operatorname{Re} \int_{0}^{1} \frac{g(t)}{\left(z^{2}-t^{2}\right)^{1 / 2}} d t .
$$

The second boundary condition of (33) is satisfied automatically and the first condition implies

$$
\int_{0}^{x} \frac{g(t)}{\left(x^{2}-t^{2}\right)^{1 / 2}} d t=T_{2 k+1}(x) \text { for } 0 \leqslant x \leqslant 1 .
$$

Hence

$$
\begin{aligned}
g(t) & =\frac{2}{\pi} \frac{d}{d t} \int_{0}^{t} \frac{x T_{2 k+1}(x)}{\left(t^{2}-x^{2}\right)^{1 / 2}} d x \\
& =\frac{2}{\pi}(2 k+1) t \int_{0}^{t} \frac{U_{2 k}(x)}{\left(t^{2}-x^{2}\right)^{1 / 2}} d x
\end{aligned}
$$


on integration by parts. Thus $g(t)$ is a polynomial of degree $2 k+1$ in $t$. By making the substitution $x=\cos (\theta / 2)$, this assumes the form of a MehlerDirichlet integral,

$$
g(t)=(2 k+1) t \frac{\sqrt{ } 2}{\pi} \int_{\phi}^{\pi} \frac{\sin \left(k+\frac{1}{2}\right) \theta}{(\cos \phi-\cos \theta)^{1 / 2}} d \theta,
$$

where $\phi=2 \cos ^{-1} t$. The Mehler-Dirichlet integral is a representation for the Legendre polynomial

$$
P_{k}(\cos \phi)=\frac{\sqrt{ } 2}{\pi} \int_{\phi}^{\pi} \frac{\sin \left(k+\frac{1}{2}\right) \theta}{(\cos \phi-\cos \theta)^{1 / 2}} d \theta
$$

and its properties are covered in Hobson [9, Chapter 18]. Hence

$$
g(t)=(2 k+1) t P_{k}\left(2 t^{2}-1\right) \text {. }
$$

On comparing the two representations for $\phi_{2 k+1}$, we see that a two-dimensional equivalent of Love's integral is

$$
R_{2 k+1}(z)=\left\{z-\left(z^{2}-1\right)^{1 / 2}\right\}^{2 k+1}=(2 k+1) \int_{0}^{1} \frac{t P_{k}\left(2 t^{2}-1\right)}{\left(z^{2}-t^{2}\right)^{1 / 2}} d t
$$

This integral representation may also be derived from other boundary-value problems of the types $C$ and $D$ but $I$ believe the above derivation is the most direct. Gladwell and England [5] have observed that $R_{n}(z)$ is a generalisation of the Chebyshev polynomials and $R_{n}(z)$ satisfies the same recurrence relation as the Chebyshev polynomials, namely

$$
R_{n+1}(z)+R_{n-1}(z)=2 z R_{n}(z) .
$$

In particular, when $n$ is even, this yields a representation for $R_{2 k}(z)$ of the form $2 z R_{2 k}(z)=\int_{0}^{1} \frac{t}{\left(z^{2}-t^{2}\right)^{1 / 2}}\left\{(2 k+1) P_{k}\left(2 t^{2}-1\right)+(2 k-1) P_{k-1}\left(2 t^{2}-1\right)\right\} d t$. Alternatively it is possible to manipulate these relations to show that

$$
R_{2 k}^{\prime}(z)=4 k \int_{0}^{1} \frac{t}{\left(z^{2}-t^{2}\right)^{1 / 2}}\left\{P_{k}^{\prime}\left(2 t^{2}-1\right)+P_{k-1}^{\prime}\left(2 t^{2}-1\right)\right\} d t .
$$

This representation can also be derived from the symmetrical case of Problem $C$ which occurs when $n=2 k$.

Various relations between Chebyshev polynomials and Legendre polynomials may be found by comparing the boundary values which occur in Problems $C$ and $D$ with the representations (42) and (43). These relations involve Abel integral equations and their inverses. It should be observed that the MehlerDirichlet integral itself may be written in the form of an Abel integral equation on a simple change of variable. For example, for Problem $C$ from (33) and (37), 
we can conclude

$$
(2 k+1) \int_{0}^{x} \frac{t P_{k}\left(2 t^{2}-1\right)}{\left(x^{2}-t^{2}\right)^{1 / 2}} d t=T_{2 k+1}(x) \text { for } 0 \leqslant x<1,
$$

and, on inversion from (20),

$$
P_{k}\left(2 t^{2}-1\right)=\frac{2}{\pi} \int_{0}^{t} \frac{U_{2 k}(x)}{\left(x^{2}-t^{2}\right)^{1 / 2}} d x .
$$

This is the Mehler-Dirichlet integral.

The symmetrical case for Problem $C$ in which $n=2 k$ yields the relation

$$
T_{2 k}^{\prime}(x)=2 k U_{2 k-1}(x)=2 \pi k A\left\{t\left[P_{k}^{\prime}\left(2 t^{2}-1\right)+P_{k-1}^{\prime}\left(2 t^{2}-1\right)\right], x\right\}
$$

from (41) and (43). This relation may be inverted using the Abel transformation relations given in Section 2.

A comparison of the separated solution with the integral representation form for various simple boundary-value problems of the types $C$ and $D$ yields the following relations:

$$
\begin{aligned}
A\left\{U_{2 k}(t), x\right\} & =P_{k}\left(2 x^{2}-1\right), \\
A\left\{t U_{2 k-1}(t), x\right\} & =\frac{1}{2}\left[P_{k}\left(2 x^{2}-1\right)+P_{k-1}\left(2 x^{2}-1\right)\right], \\
A\left\{T_{2 k}(t), x\right\} & =\frac{1}{2}\left[P_{k}\left(2 x^{2}-1\right)-P_{k-1}\left(2 x^{2}-1\right)\right]
\end{aligned}
$$

and

$$
A\left\{t T_{2 k-1}(t), x\right\}=\frac{1}{4}\left[P_{k}\left(2 x^{2}-1\right)-P_{k-2}\left(2 x^{2}-1\right)\right] .
$$

Note that, if $A\{f(t), x\}=g(x)$, then the inverse relation is

$$
f(t)=\frac{d}{d t}[A\{x g(x), t\}]
$$

Each of the relations (46) may be inverted. In particular the inverse of the first is the relation (44) (on integration). Note also that there are corresponding relations of the type (21) and inverse relations of the type (24) between these polynomials. The Abel transformations of the odd-order Chebyshev polynomials do not seem to occur naturally in these problems.

Alternative methods of solution of these problems include integral transform techniques and complex variable techniques. As in the three-dimensional problems it is possible to manipulate the Fourier transform techniques one would use to solve Problems $C$ and $D$ to give rise to Abel integral equations corresponding to (38). For Problem $C$ the Green's function approach may be used to determine the singular integral equation satisfied by $\partial \phi_{n} / \partial y=\sigma(x)$ in $|x|<1$. It is simple 
to show that $\sigma(x)$ satisfies

$$
\frac{1}{\pi} \int_{-1}^{1} \sigma(s) \log |x-s| d s=T_{n}(x) \text { for }|x| \leqslant 1 \text { and } n \geqslant 1 .
$$

This integral equation has the solution given by (34), namely $\sigma(x)=$ $-n\left(1-x^{2}\right)^{-1 / 2} T_{n}(x)$, a result which when differentiated is stated by Tricomi [14, page 180] (see also [2]). Hence alternative representations for $\phi_{n}$ are

$$
\phi_{n}=\operatorname{Re}\left\{R_{n}(z)\right\}=-\frac{n}{2 \pi} \int_{-1}^{1} \frac{T_{n}(s)}{\left(1-s^{2}\right)^{1 / 2}} \log \left[(x-s)^{2}+y^{2}\right] d s .
$$

This result is equivalent to the complex integral representation derived by Gladwell and England [5]

$$
R_{n}^{\prime}(z)=\frac{n}{\pi} \int_{-1}^{1} \frac{T_{n}(s)}{\left(1-s^{2}\right)^{1 / 2}(s-z)} d s .
$$

Alternatively, if we examine Problem $D$ and denote $\theta_{n}$ on $y=0,|x| \leqslant 1$ by $\tau(x)$, then the singular integral equation satisfied by $\tau(x)$ is

$$
\frac{1}{\pi} \int_{-1}^{1} \frac{\tau^{\prime}(s)}{s-x} d s=n U_{n-1}(x) \text { for }|x| \leqslant 1 .
$$

From (36) the solution of this equation is $\tau(s)=-\left(1-s^{2}\right)^{1 / 2} U_{n-1}(s)$. The corresponding homogeneous equation has a non-zero solution (see [5]) but this may be neglected here. The corresponding integral representation for $\theta_{n}$ is

Hence

$$
\theta_{n}=-\frac{y}{\pi} \int_{-1}^{1} \frac{\left(1-s^{2}\right)^{1 / 2} U_{n-1}(s)}{(s-x)^{2}+y^{2}} d s=\operatorname{Im}\left\{R_{n}(z)\right\}
$$

$$
R_{n}(z)=-\frac{1}{\pi} \int_{-1}^{1} \frac{\left(1-s^{2}\right)^{1 / 2} U_{n-1}(s)}{s-z} d s,
$$

a result which was also given in [5].

From a practical point of view it should be noted that the solutions of the homogeneous equations may have a direct physical interpretation. For example, if a two-dimensional frictionless punch with an end-face profile $y=f(x)$ makes complete contact with the half-plane $y \geqslant 0$ over the interval $|x| \leqslant 1$, the problem reduces to finding a plane harmonic function $\phi$ in $y \geqslant 0$ subject to the boundary conditions

$$
v=\frac{(\kappa+1)}{4 \mu} \phi=d+f(x) \text { on } y=0+\text { for }|x| \leqslant 1
$$

and

$$
\tau_{y y}=\frac{\partial \phi}{\partial y}=0 \quad \text { on } y=0+\text { for }|x|>1,
$$

in the usual notation. 
To this problem must be added the requirement that a non-zero resultant force $P$ acts on the half-plane under the punch. Hence as $\left.\int_{-1}^{1}(\partial \phi / \partial y)\right|_{y=0} d x=P$ then by Green's theorem $\int_{\Gamma}(\partial \phi / \partial n) d s=P$ where $\Gamma$ is the semicircle at infinity taken in an anticlockwise sense. Thus $\phi$ must have a logarithmic behaviour at infinity, in fact $\phi \sim(P / \pi) \log |z|$ for large $|z|$. Thus the derivative $\partial \phi / \partial x$ is harmonic in $y \geqslant 0$, tends to zero at infinity and satisfies the boundary conditions (49) differentiated with respect to $x$. Then using the solution to Problem $C$ (equation (33)) we might suppose

$$
\frac{\partial \phi}{\partial x}=\operatorname{Re} \sum_{1}^{N} \frac{1}{n} b_{n} R_{n}^{\prime}(z)
$$

where

$$
\sum_{1}^{N} b_{n} U_{n-1}(x)=\frac{4 \mu}{\kappa+1} f^{\prime}(x) \text { for }|x| \leqslant 1,
$$

but this series has not the required behaviour at infinity and to it must be added the solution of the homogeneous problem: $\partial \phi / \partial x=\operatorname{Re}(P / \pi)\left(z^{2}-1\right)^{1 / 2}$. Thus

$$
\phi=\operatorname{Re}\left[\sum_{1}^{N} \frac{1}{n} b_{n} R_{n}(z)-\frac{P}{\pi} \log \left\{R_{1}(z)\right\}\right]+\text { constant. }
$$

From (34), the contact stress is

$$
\tau_{y y}=\frac{1}{\left(1-x^{2}\right)^{1 / 2}}\left[-\sum_{1}^{N} b_{n} T_{n}(x)+\frac{P}{\pi}\right] \text { for }|x|<1 .
$$

Further discussion of these solutions and the corresponding Hilbert integral equation is given in [5]. It should be noted that similar difficulties do not arise in the symmetrical inflation of a line crack and this solution may be found directly from Problem $D$.

\section{Discussion}

The relations derived in this paper fall into three categories. There are the relations between the different forms of solution of the fundamental mixed boundary-value problems such as Love's integrals (13), (14) and the Green's function relations (31), (32) for the axi-symmetric problems together with the corresponding representations for $R_{n}(z)$ for the plane problems, namely (42), (43), (47) and (48). Alternative methods of solution will yield other relations of this type. The fundamental problems chosen were the problems which arose 
naturally from the separated solutions. The use of these methods in the solution of more general mixed boundary-value problems has been described in [10], [5], [4], [13], [12] and in [2].

The second set of relations concerns the solution of the singular integral equations which arise in these problems. For example, (29) has the solution (30), and there is a corresponding equation which may be derived from (32) for Problem $B$. For the plane problems the solutions of the Hilbert integral equation depend on Chebyshev polynomials. It is important to note that the polynomials which occur in these solutions are orthogonal polynomials with simple orthogonality relations. Hence the analytical or numerical solution of such singular integral equations is facilitated by the use of these orthogonal polynomial solutions (see [5], [12], [14] and the references given therein).

The third category is that of implicit connections between the special functions which occur in these solutions. Love's integrals imply that of necessity the Legendre polynomials satisfy the relations (25) and (26), together with their associated Abel transforms. The Mehler-Dirichlet integral and the plane separated solutions give rise to the relations (46) between the Chebyshev and Legendre polynomials.

\section{References}

[1] E. T. Copson, "On the problem of the electrified disck", Proc. Edin. Math. Soc. 8 (1947), 14-19.

[2] A. H. England, "Mixed boundary-value problems in potential theory", Proc. Edin. Math. Soc. 22 (1979), 91-98.

[3] A. H. England and A. E. Green, "Some two-dimensional punch and crack problems in classical elasticity", Proc. Camb. Phil. Soc. 59 (1963), 489-500.

[4] A. H. England and R. Shail, "Orthogonal polynomial solutions to some mixed boundaryvalue problems in elasticity, II", Quart. J. Mech. Appl. Math. 30 (1977), 397-414.

[5] G. M. L. Gladwell and A. H. England, "Orthogonal polynomial solutions to some mixed boundary-value problems in elasticity theory", Quart J. Mech. Appl. Math. 30 (1977), 175- 185.

[6] A. E. Green, "On Boussinesq's problem and penny-shaped cracks", Proc. Camb. Phil. Soc. 45 (1949), 251-257.

[7] A. E. Heins and R. C. MacCamy, "On mixed boundary-value problems for axially-symmetric potentials", J. Math. Anal. Applics. 1 (1960), 331-333.

[8] A. E. Heins, "Function theoretic aspects of diffraction theory", Electromagnetic waves, R. E. Langer ed. (Wisconsin University Press, 1962), 99-108.

[9] E. W. Hobson, The theory of spherical and ellipsoidal harmonics, (Cambridge University Press, 1931).

[10] E. R. Love, "The electrostatic field of two equal circular co-axial conducting disks", Quart. $J$. Mech. Appl. Math. 2 (1949), 428-451.

[11] G. Ia. Popov, "The contact problem of the theory of elasticity for the case of a circular area of contact", J. Appl. Math. Mech. 26 (1962), 207-225. 
[12] G. Ya. Popov, "Plates on a linearly elastic foundation (a survey)", Soviet Applied Mechanics 8 (1972), 231-242.

[13] I. N. Sneddon, Mixed boundary-value problems in potential theory, North-Holland (1966).

[14] F. G. Tricomi, Integral equations, (Interscience, 1967).

\section{Department of Theoretical Mechanics}

University of Nottingham

University Park

Nottingham NG7 2RD

United Kingdom 